\title{
Evaluation of VCAM-1 and ICAM-1 concentration and values of global tests concerning the coagulation system of patients suffering from subarachnoid haemorrage
}

\author{
Abu-Sitta Al-Drawi', Michał Wiciński², Grzegorz Grześk², Katarzyna Szadujkis-Szadurska², \\ Elżbieta Grześk², Mateusz Maciej Węclewicz², Agnieszka Michalska³ ${ }^{3}$, Anna Czeczuk ${ }^{4}$, \\ Elżbieta Huk-Wieliczuk ${ }^{4}$ \\ ${ }^{1}$ Department of Neurosurgery and Neurotraumatology, University Hospital No. 2, Bydgoszcz, Poland \\ ${ }^{2}$ Department of Pharmacology and Therapeutics, Faculty of Medicine, Collegium Medicum in Bydgoszcz, Nicolaus \\ Copernicus University, Poland \\ ${ }^{3}$ Department of Natural Sciences, Faculty of Physical Education and Sport, Biała Podlaska, Poland \\ ${ }^{4}$ Department of Physical Education, Faculty of Physical Education and Sport, Biała Podlaska, Poland
}

Al-Drawi A, Wiciński M, Grześk G, Szadujkis-Szadurska K, Grześk E, Węclewicz MM, Michalska A, Czeczuk A, Huk-Wieliczuk E. Evaluation of VCAM-1 and ICAM-1 concentration and values of global tests concerning the coagulation system of patients suffering from subarachnoid haemorrage. Ann Agric Environ Med. 2016; 23(4): 654-659. doi: 10.5604/12321966.1226862

\begin{abstract}
Ibstract
Introduction. The term 'subarachnoid haemorrhage' (SAH) stands for bleeding into the subarachnoid space, regardless of its source. It may be of primary character when the source of bleeding is situated within the subarachnoid space. Subarachnoid haemorrhage is often described as spontaneous bleeding, mainly in order to differentiate it from post-traumatic bleeding. Objective. The aim of the study was to evaluate the concentration of ICAM-1 and VCAM-1 in the blood of patients in the early phase following subarachnoid haemorrhage in terms of searching for markers useful in subarachnoid bleeding diagnostics and monitoring a patient's clinical state.

Materials and method. The study comprised 85 patients (47 women, 38 men), aged 29-81 (average 53 \pm 12 years), suffering from subarachnoid haemorrhage. The control group comprised 45 healthy people selected according to gender and age corresponding with the experimental group.

Results. The study revealed that the concentration of ICAM-1 and VCAM-1 was significantly higher in patients suffering from subarachnoid haemorrhage. Additionally, the concentration of fibrinogen decreased, aPTT was shorter and the concentration of D-dimers increased. The studied parameters did not differ with respect to the age or gender of the patients. It was stated that according to the Hunt and Hess scale, the concentration of ICAM-1 was considerably higher in the group of patients in the most severe neurological state, compared to other patients. It was also observed that the concentration of fibrinogen was significantly higher, aPTT was shorter, and the concentration of D-dimers increased in the afore-mentioned group. Conclusions. Evaluation of the concentration of adhesion molecules, as well as values of global tests concerning the coagulation system, may serve as a useful diagnostic tool for SAH.
\end{abstract}

Key words

subarachnoid haemorrhage, VCAM-1, ICAM-1

\section{INTRODUCTION}

Despite considerable progress in the diagnostics of the central nervous system within the last 10-20 years, a quick and accurate diagnosis of a stroke is still a serious challenge for clinicians. Recommended computed tomography imaging (CT) makes it possible to exclude haemorrhagic changes, but the sensitivity of this method concerning recent ischemic strokes is only $35 \%$. More modern methods of imaging, such as magnetic resonance (MR) or Perfusion CT, are much more expensive and less accessible in practice [1]. To a great extent, these hindrances led to the search for markers which could be used in the diagnostics and treatment of strokes.

Address for correspondence: Michał Wiciński, Department of Pharmacology and Therapeutics, Nicolaus Copernicus University, Collegium Medicum, M. Curie 9, 85-090 Bydgoszcz, Poland

E-mail: agnies.michalska@wp.pl

Received: 14 October 2013; accepted: 09 October 2014
An ideal marker which could be used clinically should meet numerous criteria, such as high sensitivity or the possibility of easy and quick assessment of its concentration in blood or blood plasma. Additionally, its concentration in blood should correlate with the concentration in cerebrospinal fluid and provide information concerning the level of neurological deficit. It is also significant that it should be able to make a differential diagnosis of haemorrhagic and ischemic incidents. To-date, numerous proteins have been identified which are concentrated in blood serum increase non-specifically both in haemorrhagic and ischemic strokes. It is not possible to present in brief all current directions of research on biochemical indices potentially significant in stroke diagnostics. The majority of studies concentrate on the analysis of glial and neuronal markers, such as protein S100B, glial fibrillary acidic protein (GFAP) or neuron-specific enolase (NSE). Other studies focus on the role of acute-phase protein and proinflammatory cytokines (TNFa, IL-1, IL-6, IL-8) in strokes. In enzymological diagnosis, much attention 
is still being paid to the metalloproteinase family, mainly to MMP-9, while among the elements of the coagulation system and fibrinolysis, D-dimer, thrombin activable fibrinolysis inhibitor (TAFI), type 1 plasminogen activator inhibitor (PAI-1), coagulation factors VII, VIII and von Willebrand factor (vWF), are those most focused on $[2,3,4]$.

Biochemical panels have not yet become routine methods of diagnosing a stroke. It should be expected that contemporary methods of neuroimaging supported by biochemical research will soon go beyond experimental medicine frames and provide completely new possibilities in the diagnostics and treatment of patients with a stroke.

Active endothelial protein - ICAM-1 (intercellular adhesion molecule-1). ICAM-1 (CD54) is a transmembrane adhesion molecule belonging to the immunoglobulin super-family. ICAM-1 molecules are located mainly on the luminal and lateral membrane of endothelial cells. As far as the chemical structure is concerned, ICAM-1 is a glycoprotein built from 5 different immunoglobulin-like domains, epithelial domain and those ending in the area of cytoplasm [5]. ICAM-1 comes into endothelial-leukocyte interaction through 2 types of receptors belonging to the integrin $\beta 2$ subfamily. These are receptors LFA1 (leukocyte function associated antigen) and Mac-1. Mutual interaction between adhesion molecules and leukocytes increases their affinity to endothelium and makes it possible for leukocytes to migrate through endothelial cells. Factors increasing the expression of ICAM-1 include pro-inflammatory cytokines IL-1 $\beta, T N F \alpha$ and INF $\gamma$, anoxia and allergens $[5,6]$.

In 1991 a soluble form of molecule ICAM-1, defined as sICAM-1, was isolated [7, 8]. Soluble isoforms of ICAM are released from endothelial cells and neoplasm cells. The same cytokines which increase the expression of transmembrane ICAM-1 serve as factors inducing soluble isoforms of molecules [6]. Research on mice deprived of ICAM-1 encoding gene (knockout mice) revealed that although the animals developed properly, the concentration of neutrophilia in peripheral blood increased, while the ability to activate lymphocytes decreased. However, tolerance towards septic shock increased visibly. This process may result from the disturbed correlations between immunocompetent cells and vascular endothelium [9].

VCAM-1 (vascular cell adhesion molecule-1). Vascular cell adhesion molecule-1 (VCAM-1) belongs to immunoglobulinlike adhesions with a relative molecular mass of $90-110 \mathrm{kDa}$. They can be found mainly on vascular endothelium cells in the area of luminar and lateral surfaces and are secreted in the constitutive or induced form. VCAM-1 is a significant adhesion factor of immunocompetent cells and makes it possible for inflammatory cells to infiltrate from the blood stream to the area of the brain affected by ischaemia. Similar to ICAM-1, factors increasing the expression of molecules include proinflammatory cytokines TNFa, IL-1 $\beta$ and INF $\gamma$, and anoxia [10]. Over-expression of VCAM-1 by proinflammatory cytokines is induced according to the pattern: INF $\gamma+$ TNF $\alpha>$ INF $\gamma+$ IL-1 $\beta>$ TNF $\alpha>$ INF $\gamma$. It confirms a commonly accepted property of cytokines redundancy, i.e. their ability to enhance the same biological effect through various signalling mechanisms. VCAM-1 has a structure characteristic of adhesion molecules analogous to ICAM-1. Lymphocytes adhesion to endothelial cells happens mainly through the receptor VLA-4 (very late antygen-4) belonging to the $\beta 1$ integrin subfamily [11].

\section{OBJECTIVE}

The aim of the study was to evaluate the concentration of ICAM-1 and VCAM-1 in the blood of patients in the early phase following subarachnoid haemorrhage (SAH) in terms of searching for markers useful in subarachnoid bleeding diagnostics and monitoring a patient's clinical state.

\section{MATERIALS AND METHOD}

Eighty-five patients (47 women and 38 men) with subarachnoid haemorrhage (SAH) aged $29-81$ (mean $53 \pm 12$ years) were qualified for the research. Patients were hospitalised in the Chair and Clinic of Neurosurgery and Neurotraumatology of University Hospital No. 1 and at the Neurosurgery and Neurotraumatology Ward of the University Hospital No. 2 of the Collegium Medicum in the Nicolaus Copernicus University in Bydgoszcz, Poland. The control group comprised 45 healthy people selected according to gender and age, with regard to the experimental group. Consent for the research was granted by the Local Bioethical Commission at the Collegium Medicum of the Nicolaus Copernicus University in Bydgoszcz.

In the experimental group of 85 patients with subarachnoid haemorrhage, the mean age was $53.09 \pm 11.83$ years, while in the control group the mean age of patients was significantly lower, i.e. $45.12 \pm 8.21$ years $\left(\mathrm{p}<0.0001^{*}\right)$.

In order to eliminate cerebral vasospasm, blood was sampled within 24 hours from the moment the incident occurred. Blood for examination was taken from the elbow vein with minimal venostasis into a test-tube filled with $3.2 \%$ sodium citrate.

In the low-platelet blood plasma stored in the temperature of $-86^{\circ} \mathrm{C}$ for the period not longer than 6 months, the concentration of the following parameters was marked with the use of immunoenzymatic ELISA method:

- concentration of soluble vascular cell adhesion molecule-1sVCAM-1 (Bender MedSystem) - (N: 675-1693 ng/ml); - concentration of soluble intercellular adhesion molecule-1 - ICAM-1 (Bender MedSystem) - (N: 129.9-297.4 ng/ml).

The following global coagulation tests were also performed: - fibrinogen concentration - (N: 2-4 g/l);

- D-dimer concentration - $(<500 \mu \mathrm{g} / \mathrm{l})$;

- activated partial thromboplastin time (aPTT) - (N: 26$30 \mathrm{~s})$;

- prothrombine time expressed as normalised prothrombine ratio (INR) - (N: 0.9-1.3).

Statistical analysis. Performed with the use of StatStoft STATISTICA 7.1 for Windows computer software. The changeability of the examined quantitative parameters was described with the use of median (Me) and lower (Q1) and upper (Q2) quartile. Tests on the normality of distribution of the variables revealed that it differed from the normal distribution (Shapiro-Wilk test). Therefore, the significance of differences between the compared groups was measured with the non-parametric U Mann-Whitney test. The level of 
$\mathrm{p}<0.05$ was accepted as the level of significance and marked with "*”. The determined Spearman correlation coefficients were also accepted as statistically significant at the level of $\mathrm{p}<0.05$, and also marked with “*”.

\section{RESULTS}

Figure 1 presents the comparison of ICAM-1 and VCAM-1 concentration levels in blood in the experimental and control group.

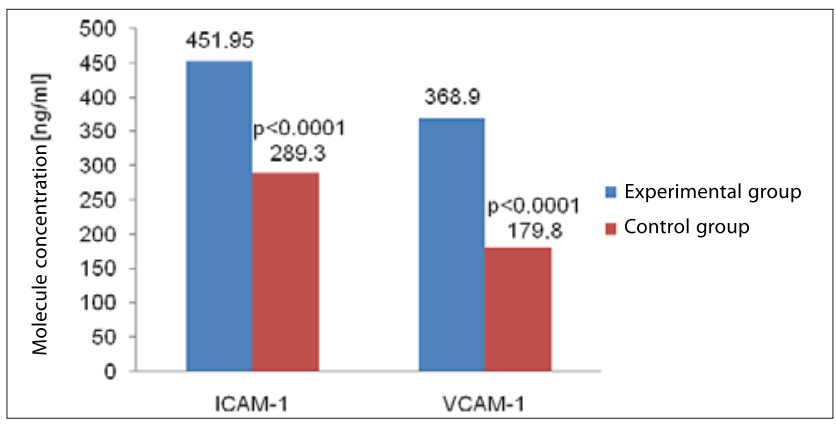

Figure 1. Comparison of ICAM-1 and VCAM-1 concentration levels in the experimental and control groups

Increased concentration levels of VCAM-1 and ICAM-1 prove the inflammatory state in patients with subarachnoid haemorrhage. The increase in the concentration levels of adhesion molecules may suggest a developing process of damaging vascular endothelium cells in the group of patients. Differences between both groups may be explained by vascular hypoperfusion, especially within the first hours after bleeding.

Table 1 shows the comparison between fibrinogen, D-dimer, INR and aPTT concentration levels in blood in the experimental and control group.

Table 1. Comparison of coagulation system parameters in the experimental and control groups

\begin{tabular}{|c|c|c|c|c|c|}
\hline \multirow[t]{2}{*}{ parameter } & \multicolumn{2}{|c|}{$\begin{array}{l}\text { experimental group } \\
\qquad \mathrm{n}=85\end{array}$} & \multicolumn{3}{|c|}{$\begin{array}{l}\text { control group } \\
n=45\end{array}$} \\
\hline & $\mathrm{Me}$ & Q1;Q3 & Me & Q1;Q3 & $\mathrm{p}$ \\
\hline $\begin{array}{l}\text { F-gen } \\
\text { [g/L] }\end{array}$ & 2.80 & $\begin{array}{l}2.10 \\
3.00\end{array}$ & 3.43 & $\begin{array}{l}3.09 \\
3.74\end{array}$ & $<0.0001^{*}$ \\
\hline INR & 0.97 & $\begin{array}{l}0.90 \\
1.00\end{array}$ & 0.92 & $\begin{array}{l}0.87 \\
0.96\end{array}$ & $0.0087^{*}$ \\
\hline $\mathrm{aPTT}[\mathrm{s}]$ & 29.00 & $\begin{array}{l}27.00 \\
30.10\end{array}$ & 32.00 & $\begin{array}{l}29.70 \\
34.20\end{array}$ & $<0.0001^{*}$ \\
\hline D-dimer $[\mathrm{ng} / \mathrm{ml}]$ & 431.00 & $\begin{array}{l}376.00 \\
803.00\end{array}$ & 167.77 & $\begin{array}{l}140.21 \\
273.11\end{array}$ & $<0.0001^{*}$ \\
\hline
\end{tabular}

What is significant is that in the group of patients with $\mathrm{SAH}$ a clear duplication of haemostasis processes can be noted. A decreased concentration of fibrinogen and increased concentration of D-dimers confirm the thesis that at the moment of bleeding the response of coagulation process dominates, which protects the structures of thecentral nervous system from damage. In the further stage, the reverse process can be seen. An increased concentration of D-dimers, i.e. the product of fibrinogen degradation, proves that there occurs a developing process of fibrinolysis. In coagulometric tests it was revealed that in the group of patients with SAH the values of INR were significantly increased. Therefore, the activity of exogenous prothrombine activation system, independent from platelets, is significantly lower in this group. The obtained medians of aPTT in patients with subarachnoid haemorrhage are lower. It seems that thrombophilia, which is often a serious clinical problem during $\mathrm{SAH}$, results from the activity of endogenous prothrombine activation system.

The results illustrate the concentration levels of ICAM-1 and VCAM-1 depending on the neurological state of the patient, assessed according to the Hunt and Hess scale (Tab. 2).

Table 2. Comparison of ICAM-1 and VCAM-1 concentration according to the Hunt and Hess scale

\begin{tabular}{|c|c|c|c|c|c|}
\hline \multirow{3}{*}{ parameter } & \multicolumn{4}{|c|}{ Hunt and Hess scale } & \multirow{3}{*}{$p$} \\
\hline & \multicolumn{2}{|c|}{$\begin{array}{l}\text { grade I-III } \\
n=66\end{array}$} & \multicolumn{2}{|c|}{$\begin{array}{c}\text { grade IV } \\
n=19\end{array}$} & \\
\hline & $\mathrm{Me}$ & Q1;Q3 & $\mathrm{Me}$ & Q1;Q3 & \\
\hline $\begin{array}{l}\text { ICAM-1 } \\
{[\mathrm{ng} / \mathrm{ml}]}\end{array}$ & 422.38 & $\begin{array}{l}348.40 \\
482.60\end{array}$ & 784.30 & $\begin{array}{l}672.10 \\
877.60\end{array}$ & $<0.0001^{*}$ \\
\hline $\begin{array}{l}\text { VCAM-1 } \\
{[\mathrm{ng} / \mathrm{ml}]}\end{array}$ & 356.63 & $\begin{array}{l}201.90 \\
550.60\end{array}$ & 378.70 & $\begin{array}{l}184.76 \\
699.92\end{array}$ & 0.8826 \\
\hline
\end{tabular}

The results shown in the Table prove that the neurological state of the patients influenced the concentration of ICAM1. In the group of 19 patients with Hunt and Hess grade IV $\mathrm{SAH}$, the ICAM-1 concentration levels were significantly higher than in the remaining patients. Statistically significant differences concerning ICAM concentration levels among the patients qualified to the 2 groups were noted between the groups of patients with grade I-III and with grade IV.

Table 3 presents the results of global coagulation system tests, i.e. the concentration of fibrinogen, D-dimer, aPTT and INR taking into consideration Hunt and Hess scale.

Table 3. Comparison of D-dimer, fibrinogen, aPTT and INR concentration according to the Hunt and Hess scale

\begin{tabular}{|c|c|c|c|c|c|}
\hline \multirow{3}{*}{ parameter } & \multicolumn{4}{|c|}{ Hunt and Hess scale } & \multirow{3}{*}{$\mathrm{p}$} \\
\hline & \multicolumn{2}{|c|}{$\begin{array}{c}\text { grade } I-I I I \\
n=66\end{array}$} & \multicolumn{2}{|c|}{$\begin{array}{c}\text { grade IV } \\
n=19\end{array}$} & \\
\hline & $\mathrm{Me}$ & Q1;Q3 & $\mathrm{Me}$ & Q1;Q3 & \\
\hline $\begin{array}{l}\text { F-gen } \\
{[\mathrm{g} / \mathrm{L}]}\end{array}$ & 2.60 & $\begin{array}{l}2.10 \\
2.90\end{array}$ & 3.90 & $\begin{array}{c}2.90 \\
4.302 \\
\end{array}$ & $<0.0001^{*}$ \\
\hline INR & 0.90 & $\begin{array}{l}0.90 \\
1.00\end{array}$ & 1.00 & $\begin{array}{l}0.90 \\
1.10\end{array}$ & 0.1065 \\
\hline aPTT [s] & 29.10 & $\begin{array}{l}27.50 \\
30.20\end{array}$ & 21.20 & $\begin{array}{l}20.30 \\
29.00\end{array}$ & $0.0002^{*}$ \\
\hline D-dimer [ng/ml] & 403.00 & $\begin{array}{l}333.00 \\
481.00\end{array}$ & 1565.00 & $\begin{array}{c}803.00 \\
5161.00\end{array}$ & $<0.0001^{*}$ \\
\hline
\end{tabular}

In the group of patients with grade IV, the concentration of fibrinogen was significantly higher, aPTT was much shorter and the concentration of D-dimer was significantly higher than in the blood of the remaining patients.

The concentration levels of ICAM-1 and VCAM-1, taking into consideration the volume of extravasated blood assessed according to the Fisher scale, are presented in Table 4.

In order to define the correlations between the concentration levels of adhesion molecules and the amount of blood which 
Table 4. Comparison of ICAM-1 and VCAM-1 concentration according to the Fisher scale

\begin{tabular}{lccccc}
\hline & \multicolumn{4}{c}{ Fisher scale } & \multirow{2}{*}{$\mathrm{p}$} \\
\cline { 2 - 5 } parameter & \multicolumn{2}{c}{ grade I-II } & \multicolumn{2}{c}{ grade III-IV } \\
& $\mathrm{n}=52$ & $\mathrm{n}=33$ & \\
\cline { 2 - 5 } & $\mathrm{Me}$ & $\mathrm{Q} 1 ; \mathrm{Q} 3$ & $\mathrm{Me}$ & $\mathrm{Q} 1 ; \mathrm{Q} 3$ & \\
\hline ICAM-1 & 404.40 & 334.99 & 633.00 & 534.20 & $<0.0001^{*}$ \\
[ng/ml] & & 452.25 & & 795.71 & \\
\hline VCAM-1 & 349.28 & 224.03 & 378.70 & 179.51 & 0.4434 \\
[ng/ml] & & 646.94 & & 539.16 & \\
\hline
\end{tabular}

got into subarachnoid space, patients with SAH were divided into 2 groups. The first group included patients with grade I/II according to the Fisher scale, while the second group included patients with grade III/IV. There were 52 patients with grade I/II and 33 patients with grade III/IV. The analysis indicated that the concentration of ICAM-1 was higher in the group of patients with grade III/IV, according to the Fisher scale, than in those with grade I/II, and the difference is statistically significant.

\section{DISCUSSION}

The research revealed higher concentration of ICAM-1, VCAM-1 and D-dimer, a significantly lower concentration of fibrinogen and shorter aPTT in blood of patients with subarachnoid haemorrhage. No differences in the examined parameters with regard to the age or gender of the patients were noted.

It was concluded that according to the Hunt and Hess scale, patients in the most serious neurological state had significantly higher concentration of ICAM-1 than the remaining patients. Moreover, in this group it was observed that 19 patients had a significantly higher concentration of fibrinogen, shorter aPTT and increased concentration of D-dimer. Analysis of the examined parameters according to the Fisher scale, taking into consideration the volume of extravasated blood, revealed the concentration of ICAM-1 in the group of patients with grade III/IV. Values of aPTT, i.e. activated partial thromboplastin time, also called kaolin-cephalin time, were calculated in the research. The results revealed significantly shorter aPTT in the group of patients with SAH. Values of aPTT were also dependent on the neurological state of the patients. Significantly shorter aPTT occurred in patients qualified for grade IV according to the Hunt and Hess scale than in patients with grade I/II, although in both groups these values fell within the norm. Shorter aPTT in patients with subarachnoid haemorrhage proved hypercoagulability resulting from the activation of intrinsic coagulation cascade.

The lower concentration of fibrinogen presumably stemmed from the increased activation of fibrinolysis by thrombin. The increase in the concentration of thrombin resulting from the activation of the coagulation system serves as a significant factor increasing the release of tissue plasminogen activator (tPA) which, after connecting with plasminogen, transforms it into plasmin. Apart from fibrin, the active proteolytic enzyme also decomposes fibrinogen. The increase in the intensity of the process of fibrinogenolysis caused by plasmin may lead to a lower concentration of fibrinogen in an early phase of subarachnoid haemorrhage. Hypofibrinogenaemia is a factor predisposing to bleeding. It seems that in patients with $\mathrm{SAH}$, the decreased concentration of fibrinogen depends on the increased fibrinogenolysis.

The research revealed a significant increase in D-dimer in patients with SAH. It is worth highlighting that this process was more visible in patients in a worse neurological state. Increase in concentration of D-dimer followed activation of fibrinolysis in an early phase of SAH.

The ICAM-1 concentration was significantly higher in patients with SAH. The results obtained in the research comply, as far as this issue is concerned, with the data published by Polin et al. in 1998. The authors of this study also described a quick increase in ICAM-1 concentration on endothelial cells already within 3 hours from the moment subarachnoid haemorrhage occurred. Also ICAM1 concentration in the cerebrospinal fluid increased until 48 hours after the beginning of the incident $[12,13,14]$. An increased concentration of sICAM-1 was noted in patients with inflammation, which indicates an increased participation of inflammatory cells, e.g. leukocytes, in this process.

A completely new perspective on the process of regulating cerebral circulation in pathological states is noted in the findings of Bavbek et al. from 1998. In this study, it was revealed for the first time that the regulation of cerebral circulation also depends on ICAM-1 adhesion molecules and their receptors LFA-1 [15]. The research carried out by Bavbek et al. revealed that the application of monoclonal antibodies directed against ICAM-1 decreases the strength of cerebral vasospasm in the course of bleeding. This is undoubted proof that ICAM/LFA-1 participates in this process. The elimination of ICAM-1 in patients after a stroke prevented pathological vasospasm in the days following the incident.

In the literature on the subject, the authors of the current study did not come across any description of the regulation of cerebral perfusion by ICAM/LFA-1 in physiological conditions. However, this unquestionably significant mechanism in the pathogenesis of vasospasm in the course of subarachnoid haemorrhage requires further research. Perhaps the application of anti-ICAM-1 antibodies will make it possible to decrease the area of cerebral anoxia and, consequently, the neurological deficit occurring during hypoperfusion. This molecule may become a significant aim of pharmacotherapy for 2 reasons. Firstly, ICAM-1 inhibition has a neuroprotective function and may decrease clinical symptoms of anoxia. Secondly, ICAM-1 may be a significant factor in the pathogenesis of vasospasm accompanying bleeding. Higher adhesion of immunocompetent cells and the fact that they release cytotoxic mediators of inflammatory reaction may lead to the intensification of the vasoconstriction effect.

Increased concentration of sICAM-1 positively correlates with the level of development of atheromatous plaques in carotid arteries, and it is a factor increasing the risk of ischemic stroke [16]. Research on the animal model of ischemia revealed that the expression of ICAM-1 in endothelial cells increases significantly already within the first hour from the incident, and remains on a higher level until the $7^{\text {th }}$ day. Additionally, a higher concentration of ICAM-1 in the cerebral ischemia focus was observed in the post-mortem examination $[16,17]$.

The increase in the concentration of ICAM-1 in the presented study may also have a certain neuroprotective 
meaning in the acute bleeding phase. It is known that the contraction of the muscular coat of cerebral arteries induced by the activation of sympathetic system, may be enforced additionally by ICAM-1 and its receptor in the area of arterial vessels. Following this path, a quick increase in the concentration of molecules may be explained as a preventive action before further blood extravasation. However, the same mechanism has an unquestionably negative meaning in the days following the bleeding since it induces recurrent vasospasms dangerous for a patient. The fact that they occur is connected with the deteriorating neurological state of the patient and usually with a high mortality rate $[18,19]$.

On the basis of the determined Spearman correlation rank values, a significant correlation between the concentration of ICAM-1 and conventionally assessed parameters of coagulation system may be noted. Positive correlation between ICAM-1 concentration and D-dimer concentration indicates an accompanying activation of fibrinolysis when blood extravasation from the ruptured aneurysm occurs, which acts to some extent as a protective mechanism preventing further neurodegeneration. Drawing on the obtained results, it may also be concluded that increased concentration of ICAM-1 and the activation of coagulation system (assessed on the basis of aPTT and fibrinogen concentration) and fibrinolysis (increased concentration of D-dimers) occur already within the first minutes after subarachnoid haemorrhage.

Increased concentration of VCAM-1 already within the first hours following SAH contributes to the development of the inflammatory state and cerebral oedema. On the other hand, Blann et al. also emphasised the meaning of this molecule in post-stroke correction mechanisms [20].

Immunocytochemical examinations of the brain of patients who died as a result of ischemic stroke revealed a significant increase in VCAM-1 concentration on the surface of endothelial cells and astrocytes, although only in the area of brain infarction $[20,21]$. Blann et al. indicated the increase in the level of plasmatic SVCAM-1 in an acute phase of the stroke in a group of patients whose blood was sampled within 12 hours from the moment the first neurological symptoms occurred. These authors also claimed that the level of plasmatic sVCAM-1 is increased until the third month from the moment the incident occurred. These results also emphasise the meaning of VCAM-1 not only in the initial phase of the stroke, where VCAM-1 enables the activation and infiltration of lymphocytes and neutrophil diapedesis in damaged brain area, but also in post-stroke correction mechanisms [20]. This indicates the significance of VCAM-1 in the chronic inflammatory state.

\section{CONCLUSIONS}

1. ICAM-1 and VCAM-1 concentration is higher in patients in an early phase of subarachnoid haemorrhage, which indicates an inflammatory state at the site where bleeding occurred, as well as the participation of immunological process.

2. Certain correlations between the clinical state of the patients, the volume of extravasated blood and the values of VCAM-1 and ICAM-1 concentration can be noted. Therefore, in the group of patients qualified for grade I-III according to the Hunt and Hess scale, significantly lower ICAM-1 values can be noted than in patients in a more severe neurological state (grade IV). No similar correlation was noted for VCAM-1 concentration. It seems significant that in patients qualified for grade I or II according to the Fisher scale, the concentration of ICAM-1 was also lower than in patients with grade III and IV. Medians of VCAM-1 values did not differ within both groups.

3. Duplication of the haemostasis processes can be noted in patients with subarachnoid haemorrhage. Shorter aPTT proves hypercoagulability resulting from the activation of intrinsic coagulation cascade, while increased concentration of D-dimers and decreased concentration of fibrinogen indicate an active fibrinogenolysis process.

4. Evaluation of the concentration levels of adhesion molecules, together with the values of global tests of coagulation system, may serve as a helpful SAH diagnostic tool.

\section{REFERENCES}

1. Kidwell CS, Chalela JA, Saver JL, Starkman S, Hill MD, Demchuk AM, et al. Comparison of MRI and CT for detection of acute intracerebral hemorrhage. JAMA 2004; 292(15): 1823-1830.

2. Anand N, Stead LG. Neuron-specific enolase as a marker for acute ischemic stroke: a systematic review. Cerebrovasc Dis. 2005; 20(4): 213-219.

3. Galis ZS, Khatri JJ. Matrix metalloproteinases in vascular remodeling and atherogenesis: the good, the bad, and the ugly. Circ Res. 2002; 90(3): 251-262.

4. Marchi N, Cavaglia M, Fazio V, Bhudia S, Hallene K, Janigro D. Peripheral markers of blood-brain barrier damage. Clin Chim Acta 2004; 342(1-2): 1-12.

5. Stolpe A, Saag PT. Intercellular adhesion molecule-1. J Mol Med. 1996; 74(1): 13-33.

6. Acevedo A, del Pozo MA, Arroyo AG, Sánchez-Mateos P, GonzálezAmaro R, Sánchez-Madrid F. Distribution of ICAM-3 bearing cells in normal human tissues. Expression of a novel counter-receptor for LFA-1 in epidermal Langerhans cells. Am J Pathol. 1993; 143(3): 774-783.

7. Rothlein R, Mainolfi EA, Czajkowski M, Marlin SD. A form of circulating ICAM-1 in human serum. J Immunol. 1991; 147(11): 3788-3793.

8. Seth R, Raymond FD, Makgoba MW. Circulatig ICAM-1 isoforms: diagnostic prospects for inflammatory and immune disorders. Lancet 1991; 338(8759): 83-84.

9. Sligh JE, Ballantyne CM, Rich SS, Hawkins HK, Smith CW, Bradley $\mathrm{A}$, et al. Inflammatory and immune responses are impaired in mice deficient in intracellular adhesion molecule-1 deficient mice. Proc Natl Acad Sci USA. 1993; 90(18): 8529-8533.

10. Shimizu Y, Newman W, Tanaka Y, Shaw S. Lymphocyte interactions with endothelial cells. Immunol Today. 1992; 13(3): 106-112.

11. Pepinsky B, Hession C, Chen LL, Moy P, Burkly L, Jakubowsky A, et al. Structure/function studies on vascular cell adhesion molecule-1. J Biol Chem. 1992; 267(25): 17820-17826.

12. Handa Y, Kabuto M, Kobayashi H, Kawano H, Takeuchi H, Hayashi $\mathrm{M}$. The correlation between immunological reaction in the arterial wall and the time course of the development of cerebral vasospasm in a primate model. Neurosurgery. 1991; 28(4): 542-549.

13. Kraus J, Gerriets T, Leis S, Stolz E, Oschmann P, Heckmann JG. Time course of VCAM-1 and ICAM-1 in CSF in patients with basal ganglia haemorrhage. Acta Neurol Scand. 2007; 116(1): 49-55.

14. Sessler CN, Windsor AC, Schwartz M, Watson L, Fisher BJ, Sugerman HJ, et al. Circulating ICAM-1 is increased in septic shock. Am J Respir Crit Care Med. 1995; 151(5): 1420-1427.

15. Bavbek M, Polin R, Kwan AL, Arthur AS, Kassell NF, Lee KS. Monoclonal antibodies against ICAM-1 and CD18 attenuate cerebral vasospasm after experimental subarachnoid hemorrhage in rabbits. Stroke. 1998; 29(9): 1930-1936.

16. Tanne D, Haim M, Boyko V, Goldbourt U, Reshef T, Matetzky S, et al. Soluble intercellular adhesion molecule-1 and risk of future ischemic stroke: a nested case-control study from the Bezafibrate Infarction Prevention (BIP) study cohort. Stroke 2002; 33(9): 2182-2186. 
17. Sobel RA, Mitchell ME, Fondren G. Intercellular adhesion molecule-1 (ICAM-1) in cellular immune reactions in the human central nervous system. Am J Pathol. 1990; 136(6): 1309-1316.

18. Khurana VG, Besser M. Pathophysiological basis of cerebral vasospasm following aneurysmal subarachnoid haemorrhage. J Clin Neurosci. 1997; 4(2): 122-131.

19. Wijdicks EF, Kallmes DF, Manno EM, Fulgham JR, Piepgras DG. Subarachnoid hemorrhage. Neurointensive care and aneurysm repair. Mayo Clin Proc. 2005; 80(4): 550-559.
20. Blann A, Kumar P, Krupinski J, McCollum C, Beevers DG, Lip GY. Soluble intercellular adhesion molecule-1, E-selectin, vascular cell adhesion molecule-1 and von Willebrand factor in stroke. Blood Coagul Fibrinolysis. 1999; 10(5): 277-284.

21. Kałuża J, Krupiński J, Kumar P, Kumar S, Wang JM. VCAM-1 expression on reactive and tumour astrocytes. Folia Histochem Cytobiol. 1994; 32(1): 17-20. 